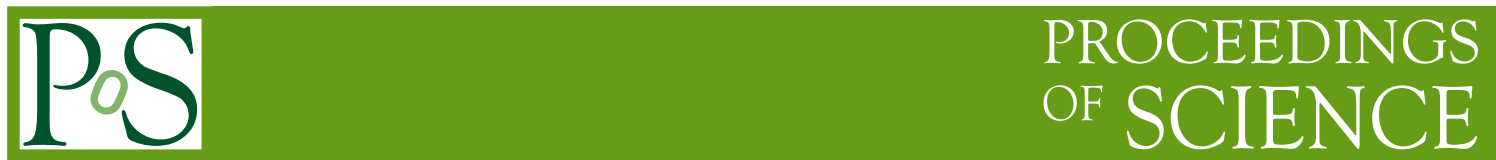

\title{
Pristine TeV cosmic-ray anisotropy in the local interstellar medium
}

\author{
Ming Zhang*t \\ Florida Institute of Technology \\ E-mail: mzhang@fit.edu

\section{Nikolai Pogorelov} \\ University of Alabama in Huntsville \\ E-mail: np0002@uah. edu
}

\begin{abstract}
We use Liouville's theorem to map cosmic-ray anisotropy from Earth back to the local interstellar medium. After taking into the account the effects of the heliosphere, we find that the pristine anisotropy of cosmic rays in the local interstellar medium inferred from Tibet AS $\gamma$ measurements at $5 \mathrm{TeV}$ is primarily a dipole due to a pitch-angle anisotropy along the interstellar magnetic field. The contributions of Compton-Getting effect, cosmic-ray density gradient and mirroring by the inhomogeneity of large-scale interstellar magnetic field are small. The dominance of dipole pitchangle anisotropy implied that particle diffusion along magnetic flux tubes is the main mechanism of cosmic-ray transport in the local interstellar medium.
\end{abstract}

35th International Cosmic Ray Conference

10-20 July, 2017

Bexco, Busan, Korea

\footnotetext{
* Speaker.

$\dagger^{\dagger}$ This work was support in part by NASA Grant NNX15AN72G.
} 


\section{Introduction}

The anisotropy in the intensity of $\mathrm{TeV}$ cosmic rays arriving at Earth is routinely measured by a number of air shower experiments, such as Tibet AS $\gamma$, IceCube, Super-Kamiokande, Milagro, ARGO-YBG, HAWC and many others ([1], [2], [3], [4], [5], [6], [7], [8], [9], [10]). With a large number of accumulated events and superior data quality, the statistics have allowed us to make a decisive determination of $\mathrm{TeV}$ cosmic-ray anisotropy and its time variation at a level better than $10^{-4}$. Observational results are quite surprising, as $\mathrm{TeV}$ cosmic-ray anisotropy patterns appear to quite complicated. There seems to be a large-scale dipole anisotropy in the order of $10^{-3}$, but its pattern is severely distorted, exhibiting small (a few degrees) to medium (tens degrees) scale angular structures. The direction of the dipole anisotropy of cosmic rays at few $\mathrm{TeV}$ energies is nearly opposite to what is expected from the motion of the solar system relative to the interstellar medium. The dipole component changes with cosmic-ray energy, and above a few hundred $\mathrm{TeV}$ its direction reverses ([6], [7], [8]). The small-scale structures in cosmic-ray anisotropy do not seem to have a correlation with any particular objects in the sky. They become more evident at lower energies around $1 \mathrm{TeV}$. These structures are often referred to as hot spots [3], [4]. Some small scale features are slightly time-dependent too (e.g., [1]).

Many attempts have been made to explain features of $\mathrm{TeV}$ cosmic-ray anisotropy using various mechanisms (e.g., [11], [12], [13], [14], [15] [16] and [17]). Among them is the theory of heliospheric contribution. The gyroradius of $1 \mathrm{TeV}$ cosmic rays in a typical interstellar magnetic field of $4 \mu \mathrm{G}$ is only $56 \mathrm{AU}$. Even at $100 \mathrm{TeV}$, the gyroradius is $5600 \mathrm{AU}$. The size of the heliosphere is a few hundred $\mathrm{AU}$ in the front direction and at least several thousand $\mathrm{AU}$ in the tail direction ([18]). The magnetic field inside the bow wave of the heliosphere is different from the original magnetic field in the local interstellar medium. When going through the heliosphere to reach Earth, the trajectories of $\mathrm{TeV}$ cosmic rays deviate from their original paths in the local interstellar field. This can cause a distortion in the angular distribution of cosmic rays arriving at Earth, just as if one looked at the sky through a frosted glass plate. In addition, there is an electric field due to the motion of heliospheric plasma relative to the local interstellar plasma. The electric field could change the energy of cosmic rays. For a measurement of cosmic rays at a fixed energy, the observed intensity will suffer an effect of particle acceleration or deceleration because of the energy dependence of source cosmic-ray spectrum.

In this paper, we are going to remove the heliospheric effects from the observed anisotropy of $\mathrm{TeV}$ cosmic rays. After presenting our theory of mapping cosmic-ray anisotropy using Liouville's theorem, we will derive a map of pristine $\mathrm{TeV}$ cosmic-ray anisotropy that we would see in the local interstellar medium without the presence of the heliosphere. Implications of this map to the behavior of cosmic ray transport and magnetic field structure in the local interstellar medium will be made.

\section{Model}

At a fixed energy, the cosmic-ray flux measured by an experiment at Earth is proportional to the particle distribution function in Earth's frame of reference. The anisotropy is just a measurement of the angular dependence of the distribution function. According to Liouville's theorem, 
the distribution function is conserved along the particle trajectory and upon Lorentz transformation of reference frames. So we can directly make the particle distribution function measured in the Earth reference frame $f\left(\mathbf{r}_{0}, \mathbf{p}_{0}\right)$ equal to that in the local interstellar medium reference frame $f(\mathbf{r}, \mathbf{p})$, where the position and momentum vectors are related along the true trajectory of any test particle. Cosmic rays are magnetized in the large-scale local interstellar magnetic field and they are sufficiently scattered by turbulence to reach the diffusion approximation with a small pitch-angle anisotropy and a small spatial density gradient. So we can always write the cosmic ray distribution function in a perturbative form, so that

$$
f\left(\mathbf{r}_{0}, \mathbf{p}_{0}\right)=f(\mathbf{r}, \mathbf{p})=f_{0} p^{-\gamma}\left[1+\nabla_{\perp} \ln f \cdot\left(\mathbf{R}_{g}-\mathbf{r}_{0}\right)+A_{\| 1} P_{1}(\mu)+A_{\| 2} P_{2}(\mu)\right]
$$

where $p$ is the magnitude of $\mathbf{p}, \mathbf{R}_{g}=\mathbf{r}-\rho_{g}$ is the location of particle guiding center that is displaced by gyroradius $\rho_{g}$ from $\mathbf{r}, P_{1}(\mu)$ and $P_{2}(\mu)$ are the first and second Legendre polynomials of particle pitch-angle cosine $\mu$ with respect to the interstellar magnetic field direction. $\gamma \approx 4.75$ is the powerlaw slope of cosmic ray distribution function. The rest are constants. $A_{\| 1}$ and $A_{\| 2}$ is the amplitudes of uni-directional and bi-directional pitch-angle anisotropy, respectively. $\nabla_{\perp} \ln f$ is the cosmicray density gradient vector perpendicular to the interstellar magnetic field. These constants are unknown parameters. They are determined from fits to observations.

Equation (2.1) depicts all the theoretical contributions to cosmic-ray anisotropy. Due to the fact that the momentum in the interstellar medium $p$ is only slightly different from the finally observed momentum $p_{0}$, or $\Delta p / p_{0}=\left(p-p_{0}\right) / p_{0} \ll 1$, a Taylor expansion essentially add one more small term $-\gamma \Delta p / p_{0}$ inside the square bracket. The dependence on the particle momentum magnitude $p$ gives rise the Compton-Getting dipole anisotropy when an observer is moving relative to the interstellar medium reference frame. It can also include the effect of particle acceleration or deceleration during cosmic-ray propagation through the heliosphere. The spatial dependence contains a second type of anisotropy, that is, the $\mathrm{b}$ cross gradient anisotropy and drift anisotropy [16]. The third type of anisotropy comes from the pitch-angle $\mu$ dependence. The pitch-angle dependence is related to cosmic-ray parallel diffusion and possible mirroring in the large-scale interstellar magnetic field. All these anisotropies are dipolar or bi-directional in a uniform magnetic field. However, because the magnetic field in presence of the heliosphere is highly nonuniform, the dipoles and bi-directional anisotropies are distorted when the particle momentum vector is mapped from the interstellar space $\mathbf{p}$ back to that at Earth $\mathbf{p}_{0}$. Medium and small-scale anisotropy features begin to show up. From equation (2.1), it can be understood that the composite anisotropy map is basically a linear combination of anisotropy maps produced by three anisotropy mechanisms. So we can use the least $\chi^{2}$ fit to observations of relative cosmic-ray fluxes to determine the free constants in Equation (2.1).

The trajectory of cosmic rays can be calculated using Newton's law with the Lorentz force in the electric and magnetic fields of the heliosphere and its surrounding. A multi-fluid MHD model is used to address the complicated charge-exchange processes between solar wind ions and interstellar neutrals and the coupling between the interstellar and heliospheric magnetic fields (see e.g., [18]). The output of the MHD model contains plasma velocity $\mathbf{V}$ and magnetic field $\mathbf{B}$. Since it is an ideal MHD model, electric field can be calculated with $\mathbf{E}=-\mathbf{V} \times \mathbf{B}$. We use time backward mapping of particle moments from the Earth location to the interstellar medium at far enough distance where we think the heliosphere influence ceases to be effective. Maps of anisotropy due to all variables 
in the distribution functions ( $p, \mathbf{R}_{g}$ and $\mu$ can be obtained once the constants $A_{\| 1}, A_{\| 2}$ and $\nabla_{\perp} \ln f$ are determined from fits to observations.

\section{Results}

\begin{tabular}{ll}
\hline Parameter & Value \\
\hline Interstellar magnetic field strength $B_{i s m}$ & $4 \mu \mathrm{G}$ \\
Interstellar magnetic field direction & see Figure 1 \\
Interstellar flow $V_{i s m}$ & $25.4 \mathrm{~km} / \mathrm{s}$ \\
Interstellar flow direction & see Figure 1 \\
Interstellar ion density $n_{p}$ & $0.0461 \mathrm{~cm}^{-3}$ \\
Interstellar neutral hydrogen density $n_{H}$ & $0.1495 \mathrm{~cm}^{-3}$ \\
Fast solar wind speed $V_{f}$ & $780 \mathrm{~km} / \mathrm{s}$ \\
Fast solar wind density at $1 \mathrm{AU} n_{f}$ & $2.2 \mathrm{~cm}^{-3}$ \\
Slow solar wind speed $V_{s}$ & $389 \mathrm{~km} / \mathrm{s}$ \\
Slow solar wind density at $1 \mathrm{AU} n_{s}$ & $7.2 \mathrm{~cm}^{-3}$ \\
Heliospheric magnetic field at $1 \mathrm{AU} B_{1 \mathrm{AU}}$ & $49 \mu \mathrm{G}$ \\
Heliospheric cirrent sheet tilt & vary from $8^{\circ}$ to $90^{\circ}$ over 11-y cycle \\
\hline
\end{tabular}

Table 1: Solar and interstellar plasma and magnetic field parameters used in MHD heliosphere simulation

\begin{tabular}{lcccc}
\hline Parameter & $A_{\| 1}$ & $A_{\| 2}$ & $\left|\nabla_{\perp} \ln f\right|$ & Direction of $\nabla_{\perp} \ln f$ \\
Value & $0.19 \%$ & $0.014 \%$ & $0.050 \% / \mathrm{R}_{g}(278 \mathrm{AU})$ & see Figure 1 \\
\hline
\end{tabular}

Table 2: Derived parameters for the local interstellar distribution function of $5 \mathrm{TeV}$ cosmic rays

For this particular calculation, we use the parameters listed in Table 1 for the MHD heliosphere model. The size of MHD simulation box in a heliocentric coordinate system is $(-10400,1600)$ AU along the axis in the direction approximate towards the inward interstellar flow, $(-2000,2058)$ AU in the direction perpendicular to the solar equatorial plane, $(-2500,2617) \mathrm{AU}$ in the third axis. The offset of the box is designed to catch the long tail that is seen to extend up to 10000 AU. Figure 1 shows show an anisotropy map of $5 \mathrm{TeV}$ cosmic-ray relative intensity (top panel), our model fits with the constants listed in Table 2 (middle panel), and a calculated anisotropy map using the right side of Equation (2.1) without the Liouville mapping trough the heliospheric fields (bottom panel). As it can be seen from the comparison between the top and middle panels, our model reproduces all the major features of the large-scale anisotropy seen observations. The cosmic-ray intensity is high in the tail direction as shown by the red areas. The red elongated area of the enhanced cosmicray intensity is approximately aligned with the plane containing the interstellar flow and magnetic field vectors, which is often referred to as the hydrogen deflection plane. The shape of the red area corresponds to a cross-section of the heliotail as viewed from Earth. The blue area has low cosmicray intensities. Its boundaries with the green areas approximately align with the plane perpendicular to the interstellar magnetic field on the right-hand side and with the bow wave (more precisely the 


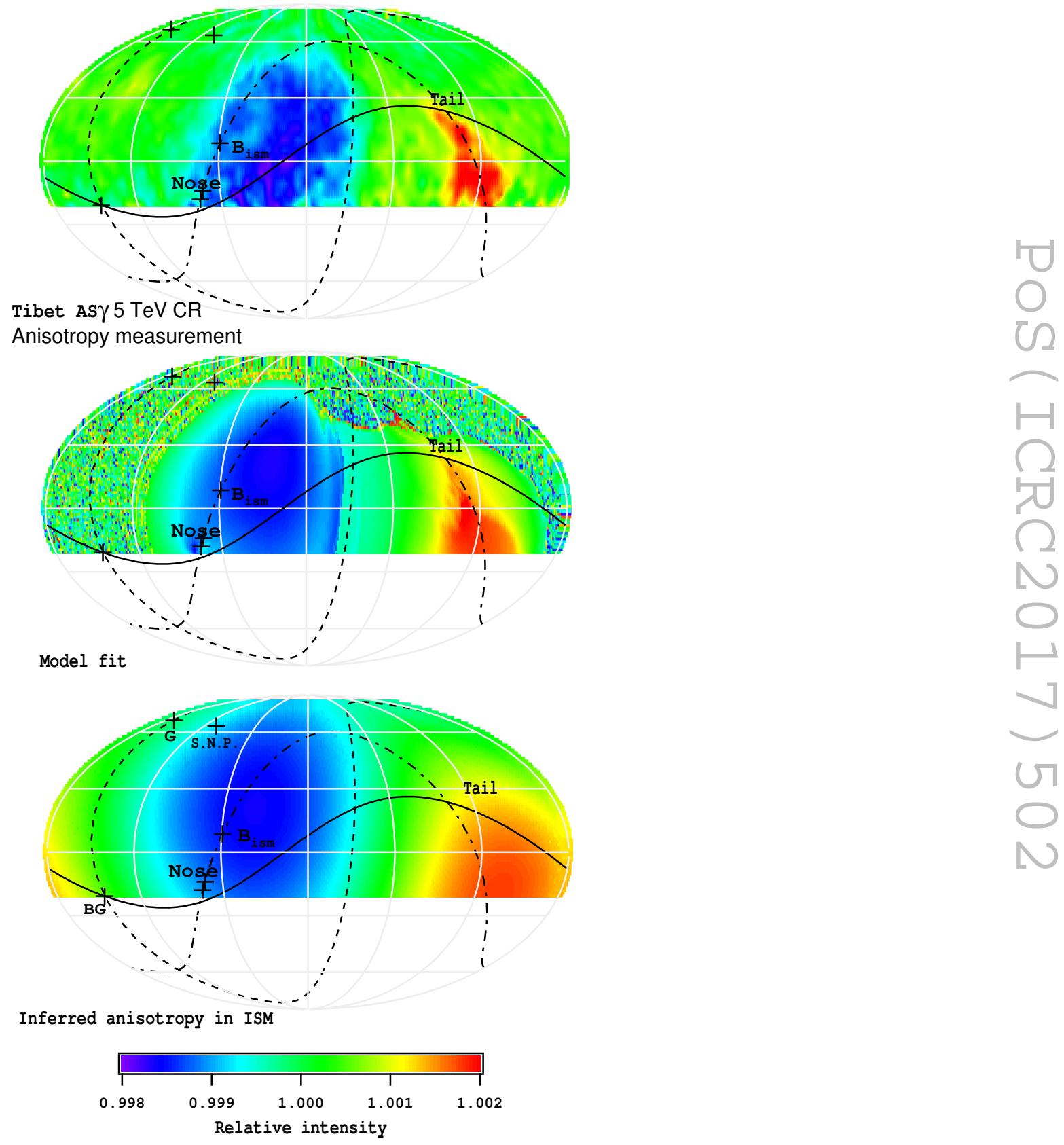

Figure 1: Tibet $\mathrm{AS} \gamma$ ([19]) measurements of $5 \mathrm{TeV}$ cosmic-ray anisotropy as a function of declination and right ascension (top), least $\chi^{2}$ fit to Tibet measurements using Liouville mapping (middle), inferred map of cosmic-ray anisotropy in the pristine interstellar anisotropy without the presence of the heliosphere (bottom). The "+" signs indicate the direction of the interstellar magnetic field $\mathbf{B}_{\text {ism }}$, the direction of perpendicular cosmic-ray density gradient $G$, the direction of North pole of the sun (S.N.P.) and the direction of b cross gradient dipole anisotropy (BG). Two additional unlabelled "+" signs indicate the inflow direction of interstellar neutral helium and hydrogen. The dot-dashed curve is the hydrogen deflection plane. The dashed curve is the plane perpendicular to the interstellar magnetic field $\mathbf{B}_{i s m}$. The solid curve is the sun's equator. The sun is placed at the nose for this particular calculation. 
outer boundary of enhanced interstellar magnetic field in the outer heliosheath) on the left-hand side. Although the overall shape of the blue regions in the top and middle panels look similar, their boundary locations slightly shift to each other, indicating that the directions of interstellar magnetic field or inflow vectors need some better estimates. The calculated cosmic ray distribution is smooth because these cosmic rays come on the nose side, where the heliosheath is thin. The observations contain some small-scale fluctuations probably due to the effect of turbulence in the interstellar medium, but it could the sun as it moves across the sky in the ecliptic plane.

The bottom panel is an estimated pristine map of $5 \mathrm{TeV}$ cosmic-ray anisotropy as we would see in the local interstellar medium without the presence of the heliosphere. It is a composite map of the Compton-Getting anisotropy due to the motion of the solar system relative to the local interstellar medium, a b cross gradient anisotropy due to the cosmic-ray density gradient, a first-order pitch-angle anisotropy, and a second-order (bi-directional) pitch-angle anisotropy. The Compton-Getting dipole anisotropy has an amplitude of $0.04 \%$ for the sun's motion at $25.4 \mathrm{~km} / \mathrm{s}$ in the local interstellar medium, and its maximum cosmic-ray intensity occurs right at the nose of the heliosphere. The amplitude of the $\mathrm{b}$ cross gradient is equal to particle gyroradius times the density gradient so it is $0.050 \%$ as listed in Table 2 without the unit of particle gyroradius. The direction of b cross gradient anisotropy (labeled as BG in Figure 1) is in the plane perpendicular to the local interstellar magnetic field direction and $90^{\circ}$ away from the cosmic ray gradient vector direction $(\mathrm{G}$ in Figure 1). Compared to the amplitudes of first-order dipole pitch-angle anisotropy $A_{\| 1}=0.19 \%$, both the Compton-Getting anisotropy and b cross gradient anisotropy are much smaller. The firstorder pitch-angle anisotropy gives cosmic-ray enhancement in the direction opposite to the local interstellar magnetic field. After some cancellation by the Compton-Getting anisotropy and $\mathrm{b}$ cross gradient anisotropy, the first-order pitch-angle anisotropy still shows its dominance, leaving a general cosmic-ray intensity enhancement along the negative interstellar magnetic field direction in the tail region and a deficit toward the interstellar magnetic field direction in the nose region. The second-order pitch angle anisotropy $A_{\| 2}$ is significantly smaller the first-order one $A_{\| 1}$. It further strength is the intensity enhancement in the tail region, but it weakens the intensity in the nose region yielding an expanded blue area in the bottom panel of Figure 1.

The pristine anisotropy of $5 \mathrm{TeV}$ cosmic rays in the local interstellar medium is dominated by the first-order dipolar pitch-angle anisotropy parallel to the local interstellar magnetic field direction. The amplitude $A_{\| 1}$ yields a parallel flux of cosmic rays relative to its density $j_{\|} / f=3 A_{\| 1} c=$ $5.7 \times 10^{-3} c$, where $c$ is the speed of light. This small particle net flow is most likely to be driven by parallel diffusion. If we assume a parallel diffusion coefficient of $10^{29} \mathrm{~cm}^{2} / \mathrm{s}$ or a parallel mean free of $10^{19} \mathrm{~cm}(\sim 3 \mathrm{pc})$, the parallel cosmic-ray density gradient $\nabla_{\|} \ln f=5.2 \times 10^{-3} \mathrm{pc}^{-1}$ or $2.6 \times 10^{-8} \mathrm{AU}^{-1}$. Compared to the magnitude of perpendicular density gradient in Table 2 or $\left|\nabla_{\perp} \ln f\right|=1.8 \times 10^{-6} \mathrm{AU}^{-1}$, the $5 \mathrm{TeV}$ cosmic-ray density gradient is mainly in the perpendicular direction. The maximum perpendicular diffusion coefficient is Bohm diffusion $\kappa_{B}=\rho_{g} c / 3$, where $\rho_{g}$ is particle gyroradius. Then the maximum perpendicular flux relative to the density $j_{\perp} / f=1.7 \times 10^{-4} c$, which is still much less than the parallel flux. Therefore, we conclude the transport of cosmic rays of $5 \mathrm{TeV}$ in the local interstellar medium is dominated by the parallel flux. The perpendicular gradient vector points in the direction of $\mathrm{G}$ labeled in Figure 1, which is roughly perpendicular to the solar equator. The fact that $A_{\| 2}$ is much less than $A_{\| 1}$ suggests that there is little mirroring of $5 \mathrm{TeV}$ cosmic rays or the magnetic field is quite uniform in the local interstellar 
medium.

\section{Conclusion}

After taking into the account the effect of heliospheric magnetic and electric fields, we find that the pristine large-scale cosmic-ray anisotropy in the local interstellar medium from inferred Tibet AS $\gamma$ measurements at $5 \mathrm{TeV}$ is consistent with a composite dipole anisotropy and a small bi-directional anisotropy. The dipole anisotropy contains contributions from the Compton-Getting effect, $\mathrm{b}$ cross gradient, and pitch-angle dipole anisotropy. The pitch-angle dipole anistropy along the local interstellar magnetic field dominates all the other effects. The local cosmic-ray flux at $5 \mathrm{TeV}$ is mainly parallel to the magnetic field, but its density gradient is mainly in the perpendicular direction. All these suggest that cosmic-ray transport in the local interstellar medium is almost completely confined in the magnetic field flux tubes and the distribution of cosmic rays are filamented although the inhomogeneity is rather small.

\section{References}

[1] M. Amenomori et al. Anisotropy and Corotation of Galactic Cosmic Rays, Science, 314 (439) 2006 [astro-ph/0610671]

[2] M. Amenomori et al. On Temporal Variations of the Multi-TeV Cosmic Ray Anisotropy Using the Tibet III Air Shower Array, Astrophys. J., 711 (119) 2010

[3] A. Abdo et al. Discovery of Localized Regions of Excess 10-TeV Cosmic Rays, Phys. Rev. Lett., 101 (221101), 2008

[4] A. Abdo et al. The Large-Scale Cosmic-Ray Anisotropy as Observed with Milagro, Astrophys. J., 698 (2121) 2009

[5] G. Guillian et al. Observation of the anisotropy of 10TeV primary cosmic ray nuclei flux with the Super-Kamiokande-I detector, Phys. Rev. D, 75, (062003) 2007

[6] R. Abbasi et al. Measurement of the Anisotropy of Cosmic-ray Arrival Directions with IceCube, Astrophys. J., 718 (L194) 2010

[7] R. Abbasi et al. Observation of Anisotropy in the Arrival Directions of Galactic Cosmic Rays at Multiple Angular Scales with IceCube, Astrophys. J., 740 (16) 2011

[8] R. Abbasi et al. Observation of Anisotropy in the Galactic Cosmic-Ray Arrival Directions at $400 \mathrm{TeV}$ with IceCube, Astrophys. J., 746, (33) 2012

[9] G. Di Sciascio and Collaboration, Observation of CR Anisotropy with ARGO-YBJ, J. Phys.: Conf. Ser., 375, (052008) 2012

[10] A. Abeysekara et al. Observation of Small-scale Anisotropy in the Arrival Direction Distribution of TeV Cosmic Rays with HAWC, Astrophys. J., 706, (108) 2014

[11] L. Drury and F. Aharonian, F. A., The puzzling MILAGRO hot spots, Astroparticle Physics, 29, (420), 2008

[12] A. Lazarian and P. Desiati, Magnetic Reconnection as the Cause of Cosmic Ray Excess from the Heliospheric Tail, Astrophys. J., 722 (188) 2010 
[13] G. Giacinti and G. Sigl, Local Magnetic Turbulence and TeV-PeV Cosmic Ray Anisotropies, Phys. Rev. Lett., 109, (071101) 2012

[14] P. Desiati and A. Lazarian Anisotropy of TeV Cosmic Rays and Outer Heliospheric Boundaries, Astrophys. J., 762, (44) 2013

[15] K. Kotera, M. Perez-Garcia and J. Silk, Strangelets and the TeV-PeV cosmic-ray anisotropies, Phys. Lett. B, 725 (196), 2013

[16] M. Zhang, P. Zuo and N. Pogorelov, Heliospheric Influence on the Anisotropy of TeV Cosmic Rays, Astrophys. J., 790 (5) 2014

[17] N. Schwadron et al. Global Anisotropies in TeV Cosmic Rays Related to the SunÕs Local Galactic Environment from IBEX, Science, 343 (988) 2014.

[18] N. Pogorelov et al., The Heliotail, Astrophys. J. Lett., 812, (L6) 2015

[19] M. Amenomori et al. Time Dependence of Loss-Cone Amplitude measured with the Tibet Air-Shower Array, Proc. 32nd ICRC, 1, (62) 2011 